\title{
Enhanced thermal and pyroelectric properties in 0-3 TGS:PVDF composites doped with graphene for infrared application
}

\author{
Xiaodong Feng*, Minqiang Wang*,*, Le Li*, Zhi Yang*, \\ Minghui Cao* and Z.-Y. Cheng ${ }^{\dagger}$ \\ *Electronic Materials Research Laboratory (EMRL) \\ Key Laboratory of Education Ministry \\ International Center for Dielectric Research (ICDR) \\ Xi'an Jiaotong University, Xi'an 710049, P. R. China \\ ${ }^{\dagger}$ Materials Research and Education Center \\ Auburn University, Auburn, AL 36849, USA \\ tmqwang@xjtu.edu.cn
}

Received 30 December 2016; Revised 25 January 2017; Accepted 30 January 2017; Published 3 March 2017

\begin{abstract}
Pyroelectric composites of triglycine sulfate (TGS)-polyvinylidene difluoride (PVDF) doped with graphene are studied. It is found that the graphene can effectively improve the polling efficiency and thermal property of the composites so that the infrared detective performance can be significantly improved. For example, by adding about $0.83 \mathrm{wt} . \%$ of graphene, the infrared detective property can be improved by more than $30 \%$. It is also found that the size of the graphene plays a critical role on the property improvement. For example, the small-sized graphene prepared by ultrasonic exfoliation (UE) method is more effective than the big-sized graphene prepared by electrochemical exfoliation (EE) method.
\end{abstract}

Keywords: Composites; polling; thermal; pyroelectric; graphene doping.

\section{Introduction}

With the demands of thermal imaging, fire detection and motion sensing from infrared detectors increasing rapidly, pyroelectric-based IR detectors, which possess advantages of wide working temperature ranging, low cost, simple structure, and easy to fabricate, ${ }^{1}$ have attracted more and more attention. Some well-known pyroelectric infrared detecting materials, such as triglycine sulfate (TGS) single crystal ${ }^{2-4}$ $\left(p=2-3.5 \times 10^{2} \mu \mathrm{C} / \mathrm{m}^{2} \mathrm{~K}\right)$, lead zirconate titanate $(\mathrm{PZT})^{5}$ $\left(p=1-1.8 \times 10^{2} \mu \mathrm{C} / \mathrm{m}^{2} \mathrm{~K}\right)$, and lithium tantalite $(\mathrm{LiTaO} 3)^{6}$ $\left(p=60 \mu \mathrm{C} / \mathrm{m}^{2} \mathrm{~K}\right)$ have been used in infrared sensor due to their high pyroelectric coefficient $p$. However, these materials are brittle and have a low formability. The polymer materials, which are flexible, can stand with high mechanic impact, and easy to process, are highly desirable for the development of IR detectors. It is widely accepted that composites are the most promising materials for meeting different requirements due to the fact that the properties of a composite can be controlled and altered through different combinations of its constituents. ${ }^{7}$ Therefore, $0-3$ composites in which a polymer matrix is filled with ceramic particles can be promising pyroelectric materials by using materials with a high pyroelectric coefficient as the filler. It is expected that the $0-3$ composites will exhibit a relative high pyroelectric coefficient with an excellent mechanical strength, formability, flexibility, and stability due to the polymer matrix. ${ }^{8}$ In this work, ferroelectric TGS single crystal, ${ }^{9}$ which has a high pyroelectric coefficient, low relative permittivity, and dielectric loss factor, is an excellent material used in infrared detective area. Therefore, TGS single crystal particles are selected as filler particles for the development of pyroelectric composites. It is well known that polymers are widely used in many areas due to its excellent mechanical properties, good chemical resistance to severe environmental stress and versatility in fabrication for a variety of forms. Among various polymers, polyvinylidene difluoride (PVDF) ${ }^{10}$ which is the best known ferroelectric polymer and exhibits the highest pyroelectric coefficient $\left(p=24-27 \mu \mathrm{C} / \mathrm{m}^{2} \mathrm{~K}\right)$ among the ferroelectric polymers, ${ }^{11}$ is selected as the polymer matrix for the composites in this work. Poling is critical for a ferroelectric material to exhibit its pyroelectric effect. The poling result of a ferroelectric material is strongly dependent on the poling method used. Therefore, the poling methods have a strong influence on the properties of the pyroelectric composites.

Regarding the poling process of ferroelectrics, the electric field has to be higher than its coercive field. The coercive field of TGS is about $300 \mathrm{~V} / \mathrm{mm}$, while that of PVDF is about $50 \mathrm{kV} / \mathrm{mm}$. However, during the poling process of $0-3$ ceramic/polymer composites, on account that the impedance of polymer is much higher than TGS particle, most of the

This is an Open Access article published by World Scientific Publishing Company. It is distributed under the terms of the Creative Commons Attribution 4.0 (CC-BY) License. Further distribution of this work is permitted, provided the original work is properly cited. 
applied electric field is taken away by the polymer matrix phase and the ceramic phases are difficult to be poled. ${ }^{12}$ As a result, the pyroelectric performance of the TGS/PVDF composites will not be good enough. Therefore, in order to improve the poling efficiency of the ceramic phase, it is essential to enhance the conductivity of the polymer phase and increase the electric field proportion shared by the ceramic phase. According to some previous reports, various conductive phases, such as graphite, ${ }^{13}$ carbon nanotube $(\mathrm{CNT})^{14}$ and graphene $^{15}$ can be used in the polymer for improving the conductivity. Among various conductive phases, graphene, whose thermal conductivity is up to $5.30 \times 10^{3} \mathrm{~W} / \mathrm{mK}^{16}$ and conductivity is also outstanding, has been certified by some researches that introducing it into the polymer can reduce the specific heat capacity of the composites considerably. ${ }^{17}$ So graphene is regarded as ideal filler due to its good electrical properties and thermal properties.

For infrared application, several figures of merit (FOM) could evaluate the contribution of the detective properties of a pyroelectric material which is used in infrared sensor. They are the current FOM $\left(\mathrm{F}_{i}\right)$, voltage FOM $\left(F_{v}\right)$, detectivity FOM $\left(F_{d}\right)^{18}$ and given by

$$
F i=\frac{p}{c}, \quad F v=\frac{p}{c \varepsilon_{0} \varepsilon r}, \quad F d=\frac{p}{c \sqrt{\left(\varepsilon_{0} \varepsilon r \tan \delta\right)}},
$$

where $p$ is pyroelectric coefficient of the material, $c$ is the specific heat capacity of the pyroelectric material, $\varepsilon_{r}$ is the relative permittivity of the sample, and $\tan \delta$ is the dielectric loss factor of the material. It can be seen that many factors have effect on the infrared detective properties of the pyroelectric composites. From Eq. (1), it can be seen that higher pyroelectric coefficient and lower specific heat capacity and dielectric constant and loss factor of the composites are beneficial to the infrared application.

In this work, a methodology for enhancing the electrical and thermal properties of composites by two different graphene doping was proposed. The TGS/Graphene/PVDF 0-3 pyroelectric composites with graphene dopant were prepared by using solvent casting method. The influences of graphene doping on the specific heat capacity, volume conductivity, dielectric properties, and pyroelectric properties were discussed in detail. Furthermore, based on these properties, the FOM of the prepared composites were also calculated for their application in infrared detecting devices. There were differences between the size, conductivity, thermal property of the ultrasonic exfoliation (UE) graphene doping and those of electrochemical exfoliation (EE) graphene doping. Therefore, the effects of two different sizes of the graphene were also discussed and compared.

\section{Experiment}

The TGS single crystal was supplied by the Institute of Materials science and Engineering, Beijing University of Technology, China. The single crystal was crushed by ball-milling and then sieved to get the TGS powders whose particle size is about $3 \mu \mathrm{m}$. The PVDF powders were purchased from Alfa Aesar, America. The two different graphenes were prepared by UE and EE technology, respectively.

The pyroelectric composites specimens with graphene doping, in which the volume of TGS tiny particles and PVDF were kept the same (Volume of TGS: Volume of PVDF=1:1), were prepared by solvent casting technique. The mass fraction of graphene doping varied from 0.0 to $1.5 \mathrm{wt} . \%$. In this method, firstly the PVDF copolymer powder was dissolved in dimethylformamide (DMF) at room temperature and then predetermined amount (0wt.\%, $0.16 \mathrm{wt} . \%, 0.33 \mathrm{wt} . \%$, 0.5 wt. $\%, 0.67$ wt. $\%, 0.83$ wt. $\%, 1.0$ wt. $\%, 1.16$ wt. $\%, 1.33$ wt. $\%, 1.50 \mathrm{wt} . \%)$ of graphene was dispersed into the solution. After 20 mins of ultrasonic agitation, a known amount of TGS tiny particles was added to the copolymer/graphene solution. In order to form homogeneous mixtures, the suspension was stirred with a magnetic stirrer for $24 \mathrm{~h}$ until the TGS particles were well dispersed into the mixtures. After vacuum defoamed at room temperature for $15 \mathrm{mins}$, the suspension was cast onto polished glass plates and baked at $80^{\circ} \mathrm{C}$ in a vacuum oven to evaporate the solvent completely. The prepared composites were about $100 \mu \mathrm{m}$ in thickness. Silver electrodes were coated on both sides of the samples for subsequent properties measurements. The TGS/Graphene/PVDF composites were poled at ambient temperature with a DC field of $20 \mathrm{kV} / \mathrm{cm}$ along the thickness direction for $30 \mathrm{mins}$.

The morphology of the surface of TGS/Graphene/PVDF 0-3 composites was observed by using scanning electron microscope (FEI QUANTA F250). The Raman spectroscopy was measured by Raman spectrometer (RENISHAW INVIAREFLEX). The volume conductivity of the samples was measured by the two-probe method with Agilent 4294A and selected the data at the point of $100 \mathrm{~Hz}$. Thermal measurements and the specific heat capacity of the samples were obtained by a differential scanning calorimeter (Netsch STA499C). The heating rate was $10^{\circ} \mathrm{C} / \mathrm{min}$ in the temperature range of $25^{\circ} \mathrm{C}$ to $70^{\circ} \mathrm{C}$ and the samples were all weighed precisely at $10 \mathrm{mg}$. The relative permittivity and dielectric loss factor as a function of temperature from $25^{\circ} \mathrm{C}$ to $60^{\circ} \mathrm{C}$ at $10 \mathrm{kHz}$ were measured using HP 4284A LCR tester equipped with a temperature chamber. The pyroelectric coefficient was observed by using the Byer-Roundy method by the measurement system that was set up by ourselves, ${ }^{19}$ the pyroelectric coefficient of the composites was calculated using Eq. (2):

$$
p=\frac{I}{a} / \frac{d T}{d t}
$$

where $I$ is the pyroelectric current, $a$ is the area of electrodes, $d T / d t$ is the rate of change of temperature.

\section{Results and Discussion}

The SEM representative micrograph of sample with two different graphene doping $(\Phi=1.0 \mathrm{wt} . \%)$ is shown in Fig. 1 . 


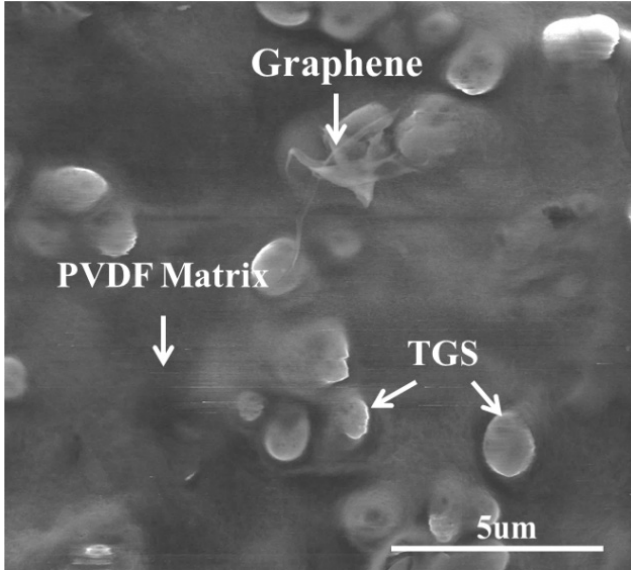

(a)

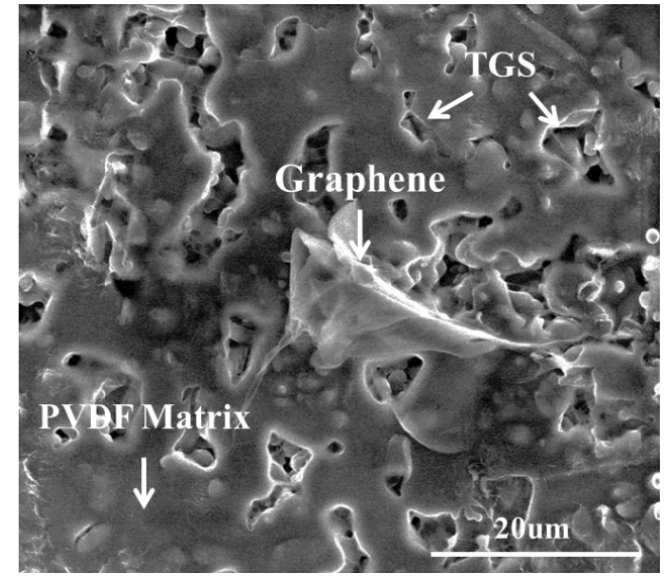

(b)

Fig. 1. (a) The SEM micrograph of sample with UE graphene doping. (b) The SEM micrograph of the sample with EE graphene doping $(\Phi=1.0$ wt. $\%)$.

It can be seen that the TGS particles were all dispersed uniformly in the PVDF matrix. The highlights of Fig. 1 were the graphene doping and it is found that the size of the graphene prepared by EE technology was much larger than that of the graphene produced by UE method.

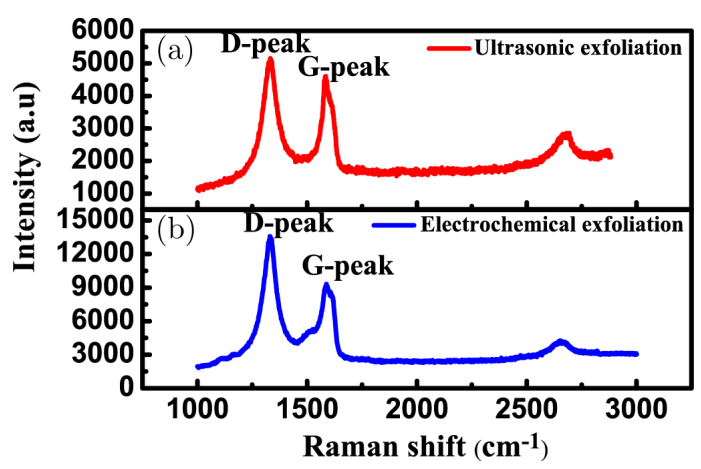

Fig. 2. The Raman spectra of the composites with the UE graphene doping (a) and EE graphene doping (b).
The Raman spectra of TGS/Graphene/PVDF 0-3 composites were shown in Fig. 2. Three major peaks: the G peak at $\sim 1580 \mathrm{~cm}^{-1}$, the D peak at $\sim 1350 \mathrm{~cm}^{-1}$ and the $2 \mathrm{D}$ peak at $\sim 2670 \mathrm{~cm}^{-1}$ was observed in the Raman spectrum. It was proved that the graphene doping have been introduced into the composites. The D-band and 2D-band were the disorder band associated with structural defects created in graphene and the G-band showed the well-ordered scattering of the $\mathrm{E}_{2 \mathrm{~g}}$ phonon of $\mathrm{sp}^{2} \mathrm{C}$ atoms of graphene. ${ }^{20}$ It can be seen that the $\mathrm{D} / \mathrm{G}$ intensity ratio of the composites with the EE graphene doping was greater than that of the composites doped with UE graphene, thus it can be concluded that the two different graphenes may make different influences on the properties of the composites.

The DSC scan of pyroelectric composites with various graphene doping contents during the heating process was plotted in Fig. 3. It can be seen that the exothermic peaks at the Curie temperature $\left(49^{\circ} \mathrm{C}\right)$ of TGS appear in the DSC curves.

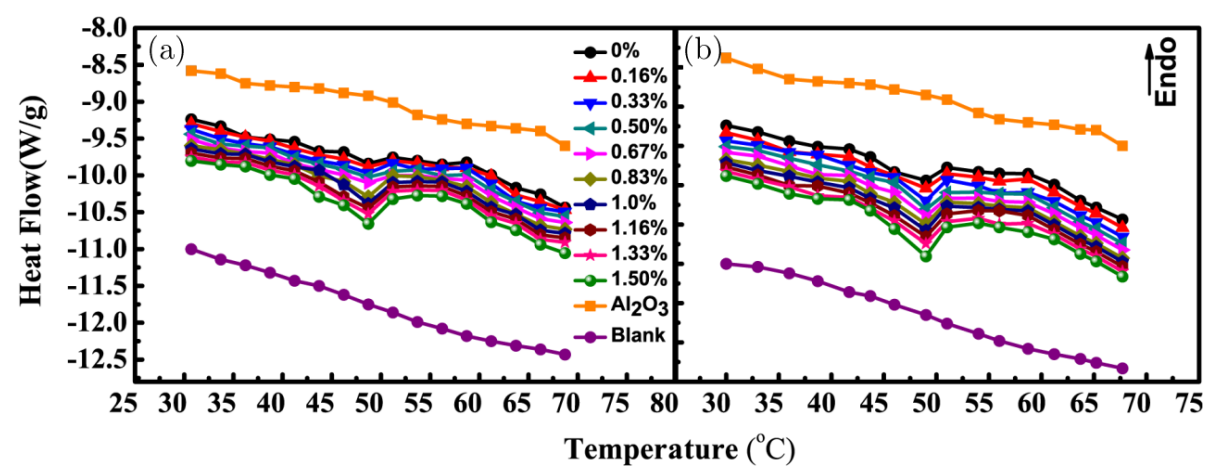

Fig. 3. The DSC scan of pyroelectric composites with the UE graphene doping (a) and EE graphene doping (b) (Volume of TGS: Volume of $\mathrm{PVDF}=1: 1$, graphene doping $\Phi=0$ wt. $\%, 0.16$ wt. $\%, 0.33$ wt. $\%, 0.50$ wt. $\%, 0.67$ wt. $\%, 0.83$ wt. $\%, 1.00$ wt. $\%, 1.16$ wt. $\%$, 1.33 wt. $\%$, $1.50 \mathrm{wt} . \%)$. 
Table 1. The specific heat capacity of pyroelectric composites with the UE graphene doping and EE graphene doping.

\begin{tabular}{|c|c|c|c|c|c|c|c|c|c|c|}
\hline Mass fraction of graphene & 0.00 wt. $\%$ & 0.16 wt. $\%$ & 0.33 wt. $\%$ & $0.50 \mathrm{wt} \%$ & 0.67 wt. $\%$ & 0.83 wt. $\%$ & $1.00 \mathrm{wt} . \%$ & 1.16 wt. $\%$ & 1.33 wt. $\%$ & 1.50 wt. $\%$ \\
\hline $\begin{array}{l}\text { The specific heat capacity } \\
\text { of the composites } \\
\text { with UE graphene } \\
\text { doping }\left(10^{6} \mathrm{~J} / \mathrm{m}^{-3} \mathrm{~K}^{-1}\right)\end{array}$ & 2.236 & 2.126 & 2.03 & 1.948 & 1.858 & 1.755 & 1.698 & 1.628 & 1.561 & 1.496 \\
\hline $\begin{array}{l}\text { The specific heat capacity } \\
\text { of the composites } \\
\text { with EE graphene } \\
\text { doping }\left(10^{6} \mathrm{~J} / \mathrm{m}^{-3} \mathrm{~K}^{-1}\right)\end{array}$ & 2.238 & 2.119 & 1.987 & 1.893 & 1.801 & 1.683 & 1.633 & 1.563 & 1.493 & 1.420 \\
\hline
\end{tabular}

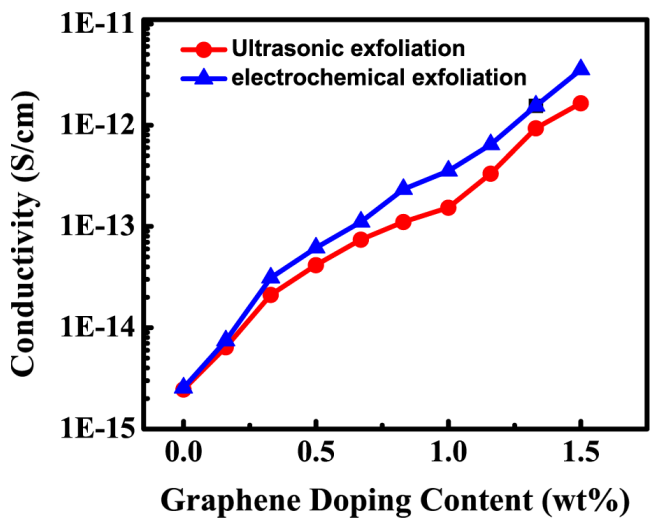

Fig. 4. The volume conductivity of PVDF polymers with the UE graphene doping and EE graphene doping (Volume of TGS: Volume of PVDF $=1: 1$, graphene doping $\Phi=0 \mathrm{wt} \%, 0.16 \mathrm{wt} . \%$, 0.33 wt. $\%, 0.50$ wt. $\%, 0.67$ wt. $\%, 0.83$ wt. $\%, 1.00$ wt. $\%, 1.16$ wt. $\%$, 1.33 wt. $\%, 1.50$ wt. $\%)$.
According to the DSC scan, the specific capacity of the composite is achieved and the results are listed in Table 1. As we can see, the specific heat capacity of pyroelectric composites decrease gradually with the increasing graphene doping content, the reason is that graphene was an excellent thermal conducting material, therefore improved the specific heat capacity of the pyroelectric composites. Besides that, it is also found that the EE graphene doping is more effective than the UE graphene doping from Table 1.

Figure 4 presents the volume conductivity of PVDF polymer as a function of graphene doping content. It was found that the volume conductivity of the pyroelectric composites improves gradually with the increase of graphene doping content and the EE graphene doping played a better result. According to Maxwell-Wagner interface mechanism, ${ }^{21}$ introducing the graphene into the PVDF matrix contributes to shortening the electronic transition distance in the PVDF and then improved the conductivity of PVDF

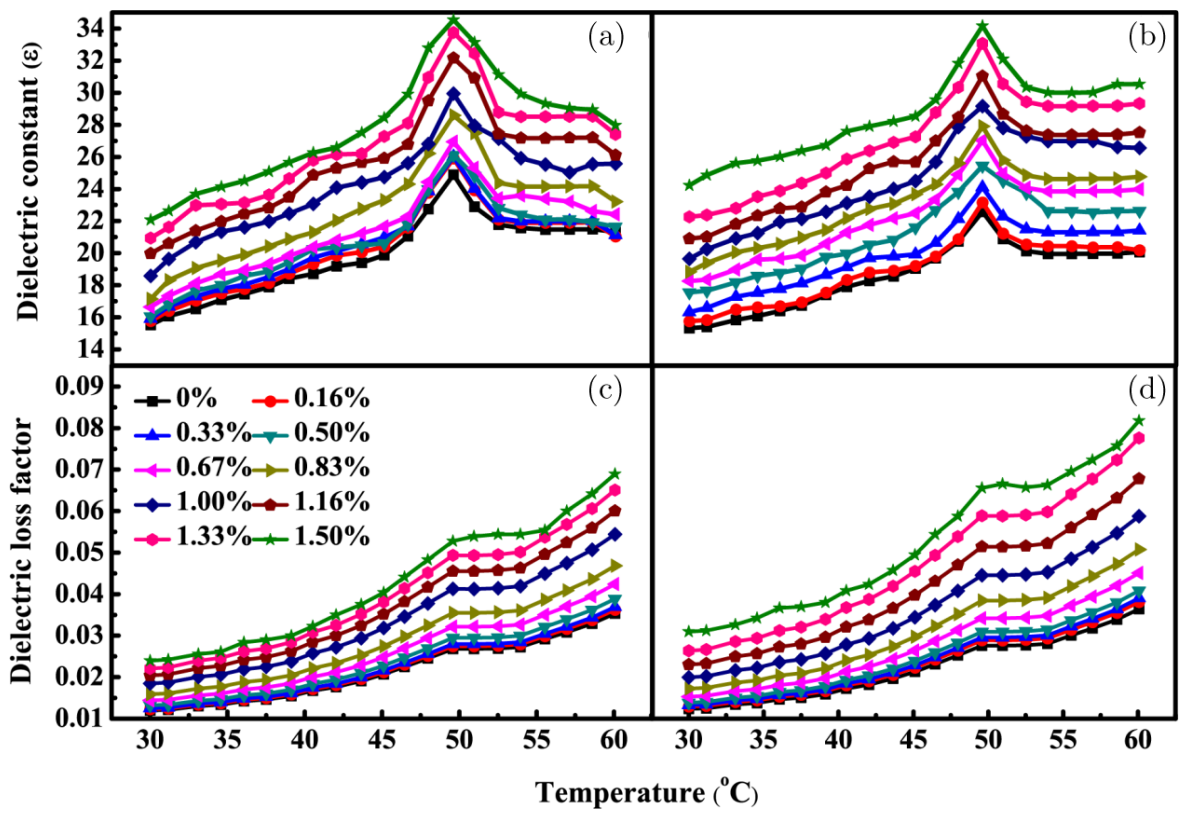

Fig. 5. The dielectric constant of the pyroelectric composites with the UE graphene doping (a) and EE graphene doping (b); the dielectric loss factor of the samples with the UE graphene doping (c) and EE graphene doping (d) (Volume of TGS:Volume of PVDF = 1:1, graphene doping $\Phi=0$ wt. $\%, 0.16$ wt. $\%, 0.33$ wt. $\%, 0.50$ wt. $\%, 0.67$ wt. $\%, 0.83$ wt. $\%, 1.00$ wt. $\%, 1.16$ wt. $\%, 1.33$ wt. $\%, 1.50$ wt. $\%$ ). 


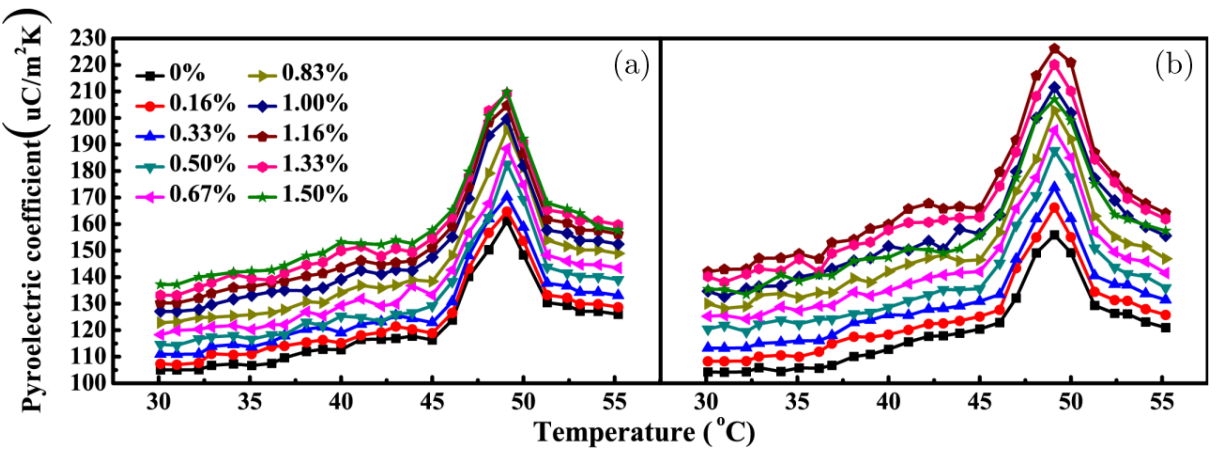

Fig. 6. The temperature dependence of pyroelectric coefficient for the composites with the UE graphene doping (a) and EE graphene doping (b) (Volume of TGS:Volume of PVDF = 1:1, graphene doping $\Phi=0$ wt. $\%, 0.16$ wt. $\%, 0.33$ wt. $\%, 0.50$ wt. $\%, 0.67$ wt. $\%, 0.83$ wt. $\%$, 1.00 wt. $\%, 1.16$ wt. $\%, 1.33$ wt. $\%, 1.50$ wt. $\%$ ).

polymer. Due to improving the conductivity of the PVDF matrix, graphene doping should be able to effectively enhance the poling efficiency of the composites.

The temperature dependence of the relative permittivity and dielectric loss factor of the 0-3 composites with graphene doping were measured at $1 \mathrm{kHz}$, and the results are shown in Fig. 5. It can be found that a peak emerged in the $\varepsilon_{r}$ versus temperature curves at the curie temperature of the TGS (about $49^{\circ} \mathrm{C}$ ). It can be seen that the dielectric constant of the composites increased with the increasing content of the graphene doping and that of the composite with EE graphene doping is higher than the composites with the same content of UE graphene doping. Besides, when the EE graphene doping content exceeds a particular amount (1.33 wt.\%), a more rapid increase of the relative permittivity emerges, which can be explained by Percolation effect. ${ }^{22}$ Obviously, the influence of graphene doping on dielectric loss factor of the pyroelectric composites was same as that of the dielectric constant in general.

Figure 6 showed the pyroelectric coefficients of the 0-3 composites with various content of graphene doping as a function of temperature from room temperature to $60^{\circ} \mathrm{C}$ (above $T_{c}$ of TGS). It was found that the addition of graphene doping can improve the pyroelectric coefficient of the composite. When the UE graphene doping content was $1.5 \mathrm{wt} . \%$ and EE graphene doping content was $1.16 \mathrm{wt} . \%$, the pyroelectric coefficients of the composites was up to about 1.4 times more than that of the composites without graphene doping. The reason was that graphene doping can improve the conductivity of PVDF matrix, thus the poling efficiency of the composites would be enhanced and then improve the pyroelectric properties of the composite. It was also found that superfluous EE graphene doping would decrease the pyroelectric coefficients of the composites. Hence, an optimum content of graphene doping should be determined.

For infrared detection application, according to Eq. (1), the excellent pyroelectric composites should possess the following merits: higher pyroelectric coefficient, lower relative permittivity, dielectric loss factor, and specific heat capacity. According to the discussion above, graphene doping can decrease the specific heat capacity and improve the pyroelectric property of the composites, it also makes the relative permittivity and dielectric loss factor increasing. What was worse, when there is too much graphene doping in the composites, the pyroelectric coefficient would decrease rapidly, reversely, the dielectric constant and dielectric loss factor of the composites would increase sharply. So in order to obtain the best infrared detective performance, it is necessary to determine a suited content of graphene doping. What is more, on account of difference in the conductivity, thermal property and size of the two different graphenes, the effects of these two graphenes on the 0-3 composites was also compared and discussed. Equation (1) was used to estimate the infrared detective property of the composites and the result was shown in Table 2.

From Table 2, it was found that when the graphene doping content is about $0.83 \mathrm{wt} . \%$, the detectivity $\operatorname{FOM}\left(F_{d}\right)$ of the pyroelectric composites was optimal, which was about 1.3 times that of those composites without graphene doping. The optimal $F_{d}$ of the composites with EE graphene doping is 43.42 and that of the composites with UE graphene doping is 45.56. This result is excellent than the research reported about

Table 2. Properties of the pyroelectric composites with various UE graphene contents.

\begin{tabular}{|c|c|c|c|c|c|c|c|c|c|c|}
\hline Mass fraction of graphene & 0.00 wt. $\%$ & 0.16 wt. $\%$ & 0.33 wt. $\%$ & 0.50 wt. $\%$ & 0.67 wt. $\%$ & 0.83 wt. $\%$ & $1.00 \mathrm{wt} . \%$ & 1.16 wt. $\%$ & 1.33 wt. $\%$ & $1.50 \mathrm{wt} . \%$ \\
\hline $\begin{array}{l}F_{d} \text { of the composites with UE } \\
\text { graphene doping }\left(10^{-6} \mathrm{~Pa}^{-1 / 2}\right)\end{array}$ & 36.5 & 38.5 & 41.18 & 42.96 & 43.72 & 45.56 & 42.92 & 42.35 & 42.15 & 41.04 \\
\hline $\begin{array}{l}F_{d} \text { of the composites with } \mathrm{EE} \\
\text { graphene doping }\left(10^{-6} \mathrm{~Pa}^{-1 / 2}\right)\end{array}$ & 35.81 & 37.83 & 39.56 & 41.75 & 42.57 & 43.42 & 42.36 & 41.21 & 40.34 & 36.23 \\
\hline
\end{tabular}


the TGS polymer composites, whose $F_{d}$ is $38.3 .^{23}$ Compared with the composites with EE graphene doping, it can be seen that the composites doped with UE graphene possessed higher detectivity FOM and showed better infrared detective performance.

\section{Conclusion}

TGS/PVDF 0-3 composites with two different graphene doping were prepared by solvent casting method. The composites were characterized by SEM, Raman spectra, and DSC. The impacts of graphene doping on the conductivity of the PVDF matrix, the specific heat capacity, dielectric and pyroelectric properties were investigated and the influences of the two different graphene doping on these properties were also discussed and compared. It was found that the $0.83 \mathrm{wt} . \%$ content of graphene doping was optimum for enhancing the electrical and thermal properties of the composites. In conclusion, graphene addition was an effective method to improve the infrared detective properties of the composites and the UE graphene was the better dopant for the composites, therefore the TGS/UE-Graphene/PVDF 0-3 composites has wide application prospect in infrared sensor.

\section{Acknowledgments}

The authors gratefully acknowledge financial support from Natural Science Foundation of China (NSFC Grant No. 51572216 and No. 61604122). This work has been financially supported by NSFC Major Research Program on Nanomanufacturing (Grant No. 91323303), the industrial science and technology research project in Shaanxi province (2015GY005), 111 Program (No. B14040) and the open projects from Institute of Photonics and Photo-Technology, Provincial Key Laboratory of Photoelectronic Technology, Northwest University, China.

\section{References}

${ }^{1}$ R. A. Dorey and R. W. Whatmore, Pyroelectric properties of PZT/ PMNZTU composite thick films, J. Electroceram. 12, 191 (2004). ${ }^{2}$ N. T. Shanthi, P. Selvarajan and C. K. Mahadevan, Growth, structural, mechanical, spectral and dielectric characterization of $\mathrm{NaCl}$-added triglycine sulfate single crystals, Curr. Appl. Phys. 9, 1155 (2009).

${ }^{3}$ J. M. Chang, A. K. Batra and R. B. Lal, Growth and characterization of doped TGS crystals for infrared devices, Cryst. Growth Des. 2, 431 (2002).

${ }^{4}$ P. M. Senthil, N. Balamurugan and V. Ganesh, Growth of TGS single crystal by conventional and SR method and its analysis on the basis of mechanical, thermal, optical and etching studies, Mater. Lett. 62, 3830 (2008).

${ }^{5}$ Q. F. Zhang, S. C. Chen, M. Y. Fan, S. L. Jiang, T. Q. Yang, J. F. Wang, G. Lia and X. Yao, Large electric-induced pyroelectric properties in Mn-doped (Pb0.87La0.02Ba0.1)(Zr0.75Sn0.16 Ti0.09)O3 ceramics, J. Alloys Compd. 547, 29 (2013).

${ }^{6}$ M. OKkuyama, Y. Togami, Y. Hamakawa, M. Kimata and S. Uematsuet, Pyroelectric infrared-CCD image sensor using LiTaO3, Sens. Actuators 16, 263 (1989).

${ }^{7}$ L. Zhang and Z.-Y. Cheng, Development of polymer-based 0-3 composites with high dielectric constant, J. Adv. Dielect. 4, 389 (2011).

${ }^{8}$ K. W. Kwok, S. T. Lau, C. K. Wong and F. G. Shin, Effects of electrical conductivity on poling of ferroelectric composites, J. Phys. D: Appl. Phys. 40, 6818 (2007).

${ }^{9}$ Y. Kim and G. Park, Pyroelectric properties of AH2PO4(A=H,K) and L-alanine doped TGS, Ferroelectrics 146, 99 (1993).

${ }^{10}$ V. T. Rathod, D. R. Mahapatra, A. Jain and A. Gayathrib, Characterization of a large-area PVDF thin film for electro-mechanical and ultrasonic sensing applications, Sens. Actuators A Phys. 163, 164 (2010).

${ }^{11}$ R. G. Kepler and R. A. Anderson, Ferroelectricity in polyvinylidene fluoride, J. Appl. Phys. 49, 1232 (1978).

${ }^{12}$ G. Sagong, A. Safari and S. J. Jang, Poling flexible piezoelectric composites, Ferroelectr. Lett. 5, 131 (1986).

${ }^{13}$ G. Ruiijanagul, S. Jompruan and A. Chaipanich, Influence of graphite particle size on electrical properties of modified PZTpolymer composites, Curr. Appl. Phy. 8, 359 (2008).

${ }^{14}$ S. Gong, Z. H. Zhu, E. I. Haddad, S. Gong, Z. H. Zhu and E. I. Haddad, Modeling electrical conductivity of nanocomposites by considering carbon nanotube deformation at nanotube junctions, J. Appl. Phys. 114, 1 (2013).

${ }^{15}$ J. H. Du and H. M. Cheng, The fabrication, properties, and uses of Graphene/Polymer composites, Macromol. Chem. Phys. 213, 1060 (2012)

${ }^{16}$ A. A. Balandin, S. Ghosh and W. Z. Bao, Superior thermal conductivity of single-layer graphene, Nano. Lett. 8, 902 (2008).

${ }^{17}$ H. Liem and H. S. Choy, Superior thermal conductivity of polymer nanocomposites by using graphene and boron nitride as fillers, Solid State Commun. 163, 41 (2013).

${ }^{18}$ A. K. Batra, M. D. Aggarwal, M. E. Edwards and A. Bhalla, Present status of polymer: Ceramic composites for pyroelectric infrared detectors, Ferroelectrics. 366, 84 (2008).

${ }^{19}$ R. L. Byer and C. B. Roundy, Pyroelectric coefficient direct measurement technique and application to a nsec response time detector, Ferroelectrics 3, 333 (1972).

${ }^{20}$ A. Gupta, G. Chen, P. Joshi, S. Tadigadapa and P. C. Eklund, Raman scattering from high-frequency phonons in supported n-graphene layer films, Nano. Lett. 6, 2667 (2006).

${ }^{21}$ R. L. Blythe, Electrical Properties of Polymer, 1st edn. (Cambridge University Press, London, 1979).

${ }^{22}$ J. W. Essam, Percolation theory, Rep. Prog. Phys. 43, 833 (1980).

${ }^{23}$ Y. Yang, H. L. W. Chan and C. L. Choy, Properties of triglycine sulfate/poly(vinylidenefluoride-trifluoroethylene) 0-3 composites, J. Mater. Sci. 41, 251 (2006). 